\title{
Turnpike and dissipativity properties in dynamic real-time optimization and economic MPC
}

\author{
Timm Faulwasser ${ }^{1}$, Milan Korda ${ }^{1}$, Colin N. Jones ${ }^{1}$ and Dominique Bonvin ${ }^{1}$
}

\begin{abstract}
We investigate the turnpike and dissipativity properties of continuous-time optimal control problems. These properties play a key role in the analysis and design of schemes for dynamic real-time optimization and economic model predictive control. We show in a continuous-time setting that dissipativity of a system with respect to a steady state implies the existence of a turnpike at this steady state and optimal stationary operation at this steady state. Furthermore, we investigate the converse statements: We show that the existence of a turnpike at a steady state implies (a) that this steady state is the optimal steady state; and (b) that over an infinite horizon the system is optimally operated at this steady state. We draw upon a numerical example to illustrate our findings.
\end{abstract}

Index Terms-dissipativity, turnpike property, dynamic realtime optimization, economic model predictive control

\section{INTRODUCTION}

Recently there has been a growing interest in dynamic real-time optimization (dRTO) under the new label economic $M P C$ (eMPC), cf. [4, 6, 11, 14, 17]. Dissipativity properties have shown to be very useful in the analysis of convergence and stability properties of dRTO/eMPC schemes $[1,4,6$, 11]. For instance, it is shown in [1] in a discrete-time setting that a dissipativity property (with respect to the supply rate related to the stage cost and an optimal steady state) implies that the system is optimally operated at this steady state over an infinite horizon. Additionally, observations of turnpike behavior of optimal control problems arising from eMPC formulations has been observed [6, 17].

The main goal of this paper is to analyze the relation between dissipativity properties, turnpike properties and optimal operation at steady state. In contrast to previous works $[1,6]$, we work in a continuous-time setting. We extend a discrete-time result on the relation between dissipativity and optimal operation at a steady state given in [1] to the continuous-time setting. Furthermore, we show that a certain dissipativity property implies the existence of a turnpike in the solutions of the related optimal control problems. In essence, we generalize a classical result on the existence of turnpike properties of optimal control problems [2] by replacing convexity assumptions and terminal constraints with dissipativity properties. Additionally, we investigate a converse statement: Assuming a purely state-dependent cost function, we show that the existence of a turnpike at a steady state implies that the system is optimally operated at this steady state. By investigating the relation between

\footnotetext{
${ }^{1}$ All authors are with the Laboratoire d'Automatique, Ecole Polytechnique Fédérale de Lausanne, CH-1004 Lausanne, Switzerland \{timm.faulwasser, milan.korda, colin.jones, dominique.bonvin\}@epfl.ch
}

dissipativity, turnpikes and optimal operation at steady state, we show that optimal operation at steady state can be verified by establishing either dissipativity or turnpike properties of the corresponding optimal control problems. Specifically, we consider a Van de Vusse reactor to demonstrate that dissipativity properties can be verified via sum-of-squares techniques.

The remainder of this paper is structured as follows: Section II introduces a formal definition of turnpike and dissipativity properties as well as the definition of optimal operation at a steady state. Section III presents the main results and their proofs. We illustrate our findings via a numerical example in Section IV.

\section{Preliminaries}

We briefly recall the notions of optimal operation at steady state, dissipativity with respect to a steady state and turnpike properties of optimal control problems.

\section{A. Optimal Steady State Operation}

We consider the nonlinear system given by

$$
\dot{x}=f(x, u), \quad x(0)=x_{0},
$$

where the states $x \in \mathbb{R}^{n_{x}}$ and the inputs $u \in \mathbb{R}^{n_{u}}$ are constrained to lie in the compact sets $\mathcal{X} \subset \mathbb{R}^{n_{x}}$ and $\mathcal{U} \subset$ $\mathbb{R}^{n_{u}}$. We assume that the vector field $f: \mathbb{R}^{n_{x}} \times \mathbb{R}^{n_{u}} \rightarrow \mathbb{R}^{n_{x}}$ is Lipschitz on $\mathcal{X} \times \mathcal{U}$. A solution of (1) starting at time 0 at $x_{0}$ driven by the input $u:[0, \infty) \rightarrow \mathcal{U}$ is denoted as $x\left(\cdot, x_{0}, u(\cdot)\right)$.

Consider the largest controlled invariant set $\mathcal{X}_{0} \subseteq \mathcal{X}$ given by

$$
\begin{aligned}
\mathcal{X}_{0}=\left\{x_{0} \in \mathcal{X} \mid \exists u:[\right. & 0, \infty) \rightarrow \mathcal{U}: \\
& \left.\forall t \geq 0 x\left(t, x_{0}, u(\cdot)\right) \in \mathcal{X}\right\} .
\end{aligned}
$$

This set is the largest subset of $\mathcal{X}$ that can be rendered positively invariant via an admissible control $u(\cdot)$.

Furthermore, consider a finite-horizon optimal control problem (OCP) that aims at minimizing the objective functional

$$
J_{T}\left(x_{0}, u(\cdot)\right)=\int_{0}^{T} F(x(t), u(t)) d t
$$

where $F: \mathcal{X} \times \mathcal{U} \rightarrow \mathbb{R}$ is the cost function and $T$ is called the optimization horizon. We assume that $F$ is Lipschitz on $\mathcal{X} \times \mathcal{U}$. The OCP reads

$$
\underset{u(\cdot) \in \mathcal{M}([0, T], \mathcal{U})}{\operatorname{minimize}} J_{T}\left(x_{0}, u(\cdot)\right)
$$


subject to (1) and

$$
\forall t \in[0, T]: \quad x(t) \in \mathcal{X}, \quad u(t) \in \mathcal{U},
$$

where $\mathcal{M}([0, T], \mathcal{U})$ denotes the class of measurable functions on $[0, T]$ taking values in the compact set $\mathcal{U} \subset \mathbb{R}^{n_{u}}$. The pair $\left(x\left(\cdot, x_{0}, u(\cdot)\right), u(\cdot)\right)$ is called admissible if $u(\cdot) \in$ $\mathcal{M}([0, T], \mathcal{U})$ and, for all $t \in[0, T]$, the corresponding trajectory satisfies $x\left(t, x_{0}, u(\cdot)\right) \in \mathcal{X}$. An optimal solution to (4) is denoted by $u^{\star}(\cdot)$ and the corresponding state trajectory is written as $x^{\star}\left(\cdot, x_{0}, u^{\star}(\cdot)\right)$. $^{1}$

While OCP (4) aims at optimizing the transient performance of system (1), one can as well ask for the best stationary operating conditions. These conditions are given by the following steady-state optimization problem:

$$
\begin{aligned}
& \underset{(\bar{x}, \bar{u}) \in \mathbb{R}^{n_{x}+n_{u}}}{\operatorname{minimize}} F(\bar{x}, \bar{u}) \\
& \text { subject to } \\
& \quad f(\bar{x}, \bar{u})=0 \\
& (\bar{x}, \bar{u}) \in \mathcal{X} \times \mathcal{U} .
\end{aligned}
$$

Here $F: \mathcal{X} \times \mathcal{U} \rightarrow \mathbb{R}$ is the same cost function as in (3). Note that throughout this paper we use the superscript - to indicate that a variable is a steady state. An optimal solution to this static optimization problem is denoted as $\left(\bar{x}^{\star}, \bar{u}^{\star}\right)$. The set of admissible steady states is denoted as

$$
\overline{\mathcal{X}}=\{\bar{x} \in \mathcal{X} \mid \exists \bar{u} \in \mathcal{U}, 0=f(\bar{x}, \bar{u})\} .
$$

The set of optimal steady states is then denoted by $\overline{\mathcal{X}}^{\star}$, i.e., $\overline{\mathcal{X}}^{\star}=\left\{\bar{x}^{\star} \in \overline{\mathcal{X}} \mid \exists \bar{u}^{\star} \in \mathcal{U}\right.$ s.t. $\left(\bar{x}^{\star}, \bar{u}^{\star}\right)$ is optimal in $\left.(5)\right\}$.

In the operation of dynamic processes, it is of major interest to know whether the best infinite horizon performance can be achieved at the best steady state or via permanent transient operation. The optimal operation over an infinite horizon is defined similar to [1, 6] as follows:

Definition 1 (Optimal operation at steady state):

System (1) is said to be optimally operated at steady state if there exists $(\bar{x}, \bar{u}) \in \overline{\mathcal{X}} \times \mathcal{U}$ such that, for any initial condition $x_{0} \in \mathcal{X}$ and any infinite-time admissible pair $\left(x\left(\cdot, x_{0}, u(\cdot)\right), u(\cdot)\right)$, we have

$$
\liminf _{T \rightarrow \infty} \frac{1}{T} J_{T}\left(x_{0}, u(\cdot)\right) \geq F(\bar{x}, \bar{u}) .
$$

The following lemma follows trivially from the former definition.

Lemma 1: If system (1) is optimally operated at the steady state $(\bar{x}, \bar{u})$, then $(\bar{x}, \bar{u})$ is an optimal solution to (5).

\section{B. Turnpike Properties of OCPS}

It is well known that the properties of infinite-horizon optimal control problems are closely related to turnpike properties; see [2, 18].

Definition 2 (Turnpike property): The solution pairs $\left(x^{\star}\left(\cdot, x_{0}, u^{\star}(\cdot)\right), u^{\star}(\cdot)\right)$ of (4) are said to have an

\footnotetext{
${ }^{1}$ Here, we assume for simplicity that the optimal solution exists and is attained. We refer to $[10,16]$ for conditions ensuring the existence of optimal solutions to OCP (4).
}

approximate turnpike property with respect to the steady state $\bar{x} \in \overline{\mathcal{X}}$ if there exists a function $\nu:(0, \infty) \rightarrow[0, \infty)$ such that for all $x_{0} \in \mathcal{X}_{0}$ and all $T>0$ we have

$$
\mu\left[\Theta_{\varepsilon, T}\right]<\nu(\varepsilon) \quad \forall \varepsilon>0,
$$

where $\mu[\cdot]$ is the Lebesgue measure on the real line and

$$
\Theta_{\varepsilon, T}:=\left\{t \in[0, T]:\left\|x^{\star}\left(t, x_{0}, u^{\star}(\cdot)\right)-\bar{x}\right\|>\varepsilon\right\} .
$$

The turnpike property states that, for any initial condition $x_{0}$ and any horizon length $T>0$, the time that the optimal solutions spend outside an $\varepsilon$-neighborhood of $\bar{x}$ is bounded by $\nu(\varepsilon)$, where $\nu(\varepsilon)$ is not a function of the horizon length $T$. Note that, according to Definition 2, the steady state at which the turnpike takes place has to be the same for all horizon lengths $T \geq 0$. In essence, the turnpike property of solutions to an OCP can be understood as the existence of an arc along which the optimal trajectory $x^{\star}(\cdot)$ stays close to the steady state $\bar{x}$ in the sense of the Euclidean norm $\|\cdot\|$, and the time length of this arc increases with increasing horizon length $T$. Similar definitions of turnpike properties for the case of discrete-time optimal control problems are used [3, 18]. For continuous-time problems, a similar definition is implicitly given in [2].

Remark 1: Note that Definition 2 implies that the steady state $\bar{x}$ at which the turnpike occurs is asymptotically reachable from all $x_{0} \in \mathcal{X}_{0}$.

\section{Dissipativity}

It has been shown in [1] that for discrete time systems a certain dissipativity property is closely linked to the question of optimal operation at steady state. We briefly recall the necessary definitions.

For some $\bar{x} \in \overline{\mathcal{X}}$ consider $w: \mathcal{X} \times \mathcal{U} \rightarrow \mathbb{R}$

$$
w(x, u)=F(x, u)-F(\bar{x}, \bar{u})
$$

where $F$ is the cost function in (3) and (5).

Definition 3 (Dissipativity with respect to a steady state): System (1) is said to be dissipative on $\mathcal{D} \subseteq \mathcal{X} \times \mathcal{U}$ with respect to the steady state $\bar{x} \in \overline{\mathcal{X}}$ if there exists a bounded storage function $S: \mathcal{X} \rightarrow \mathbb{R}$ such that

$$
\frac{\partial S}{\partial x} f(x, u) \leq w(x, u)
$$

holds for all $(x, u) \in \mathcal{D} .^{2}$ If in addition for some class $\mathcal{K}$ function $\alpha$ it holds that

$$
\frac{\partial S}{\partial x} f(x, u) \leq-\alpha(\|x-\bar{x}\|)+w(x, u),
$$

then $(1)$ is called strictly dissipative on $\mathcal{D}$ with respect to the steady state $\bar{x}$.

Due to this dissipativity notion, the function $w$ in (9) is called a supply rate.

\footnotetext{
${ }^{2}$ Note that we define the storage function $S: \mathcal{X} \rightarrow \mathbb{R}$ to take real values instead of postive real values. Since the domain of $S$ is assumed to be compact, one could as well consider $S: \mathcal{X} \rightarrow \mathbb{R}_{0}^{+}$.
} 


\section{MAIN RESULTS}

The main purpose of this paper is to establish links between the three following formal statements/assumptions:

Statement 1: System (1) is strictly dissipative on the set $\mathcal{X} \times \mathcal{U}$ with respect to the steady state $\bar{x} \in \overline{\mathcal{X}}$ and the supply rate $w(9)$.

Statement 2: System (1) is optimally operated at the optimal steady state pair $\left(\bar{x}^{\star}, \bar{u}^{\star}\right), \bar{x}^{\star} \in \overline{\mathcal{X}}^{\star}$.

Statement 3: For all $x_{0} \in \mathcal{X}_{0}$, the optimal solutions of OCP (4) have an approximate turnpike at $\bar{x}$.

Subsequently, we show that

- Statement $1 \Rightarrow$ Statement 3;

- Statement $1 \Rightarrow$ Statement 2;

- Statement $3 \Rightarrow$ Statement 2 .

These relations are sketched in Fig. 1.

For some of these relations we will invoke a reachability assumption. Consider the set

$$
\begin{aligned}
\mathcal{R}(\tilde{x}, T):= & \left\{x_{0} \in \mathcal{X} \mid \exists u(\cdot) \in \mathcal{M}([0, T], \mathcal{U}):\right. \\
& \left.x\left(\cdot, x_{0}, u(\cdot)\right) \in \mathcal{X}, x\left(T, x_{0}, u(\cdot)\right)=\tilde{x}\right\}
\end{aligned}
$$

which is the set of initial conditions $x_{0} \in \mathcal{X}$ for which a point $\tilde{x} \in \mathcal{X}$ is reachable in an admissible way in some finite time $T \in(0, \infty)$.

Assumption 1: There exists a time $T_{\overline{\mathcal{X}}^{\star}}<\infty$ such that, for all $x_{0} \in \mathcal{X}_{0}$, there exists an optimal steady state $\bar{x}^{\star} \in \overline{\mathcal{X}}^{\star}$ reachable in time $T_{\overline{\mathcal{X}}^{\star}}$, i.e.,

$$
\bigcup_{\bar{x}^{\star} \in \overline{\mathcal{X}}^{\star}} \mathcal{R}\left(\bar{x}^{\star}, T_{\overline{\mathcal{X}}^{\star}}\right) \supset \mathcal{X}_{0} .
$$

\section{Implications of Dissipativity}

We first prove a technical statement.

Lemma 2: If Statement 1 is true for some steady state $\bar{x} \in \overline{\mathcal{X}}$, then $\bar{x}=\bar{x}^{\star}$, i.e., $\bar{x}$ is the unique solution to (5) in the sense that there exists $\bar{u}$ such that $(\bar{x}, \bar{u})$ attains the optimum in (5) and $\bar{x}$ is unique.

Proof: By definition of $\bar{x}$, there exists $\bar{u} \in \mathcal{U}$ such that $f(\bar{x}, \bar{u})=0$. Let $\left(\bar{x}^{\star}, \bar{u}^{\star}\right)$ be an optimal solution to (5). Evaluating the strict dissipativity condition (11) at $\left(\bar{x}^{\star}, \bar{u}^{\star}\right)$ yields

$$
0 \leq-\alpha\left(\left\|\bar{x}^{\star}-\bar{x}\right\|\right)+F\left(\bar{x}^{\star}, \bar{u}^{\star}\right)-F(\bar{x}, \bar{u}) .
$$

Since $(\bar{x}, \bar{u})$ is feasible in (5), we have

$$
F\left(\bar{x}^{\star}, \bar{u}^{\star}\right)-F(\bar{x}, \bar{u}) \leq 0 .
$$

Thus the fact that $\alpha$ is of class $\mathcal{K}$ implies that $\bar{x}^{\star}=\bar{x}$.

Remark 2: Note that it is possible to have two steady state pairs $\left(\bar{x}, \bar{u}_{1}\right),\left(\bar{x}, \bar{u}_{2}\right)$ with $F\left(\bar{x}, \bar{u}_{1}\right)=F\left(\bar{x}, \bar{u}_{2}\right), u_{1} \neq u_{2}$ and to be dissipative with respect to $\bar{x}$.

Theorem 1 (Dissipativity $\Rightarrow$ turnpike): Suppose that Statement 1 and Assumption 1 hold for $\bar{x}^{\star} \in \overline{\mathcal{X}}$. Then Statement 3 holds at $\bar{x}^{\star}$, i.e., the optimal solutions to (4) have an approximate turnpike at $\bar{x}^{\star}$.

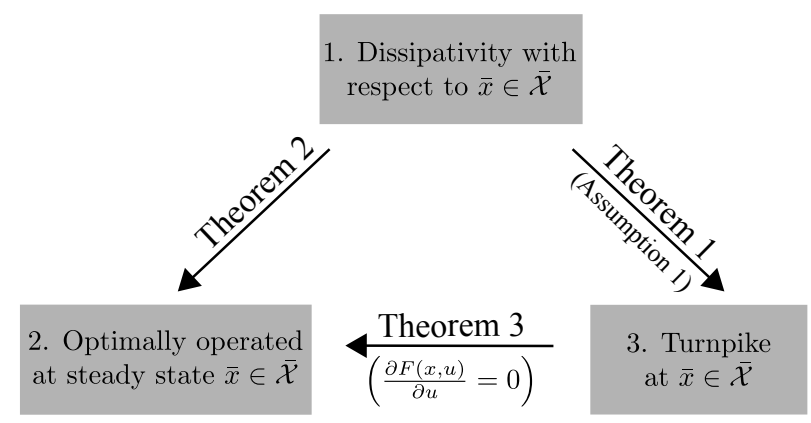

Fig. 1. Implications between disspativity, turnpike propterties and optimal operation at steady state.

Proof: Let $\left(x^{\star}(\cdot), u^{\star}(\cdot)\right)$ be an optimal solution to (4) on the horizon $[0, T]$. Integrating the dissipation inequality (11) gives

$$
\begin{aligned}
S\left(x^{\star}(T)\right)-S\left(x^{\star}(0)\right) & \leq-\int_{0}^{T} \alpha\left(\left\|x^{\star}(t)-\bar{x}^{\star}\right\|\right) d t \\
& +\int_{0}^{T} F\left(x^{\star}(t), u^{\star}(t)\right)-F\left(\bar{x}^{\star}, \bar{u}^{\star}\right) d t .
\end{aligned}
$$

By Assumption $1, \bar{x}^{\star}$ is reachable from every $x_{0} \in \mathcal{X}_{0}$ in a time $T_{\overline{\mathcal{X}}^{\star}}<\infty$; therefore, the second integral is bounded from above by $K_{F}:=2 T_{\overline{\mathcal{X}}}$ $\sup \{F(x, u) \mid x \in \mathcal{X}, u \in$ $\mathcal{U}\}<\infty$ for all $x_{0} \in \mathcal{X}_{0}$, independently of $T$. In addition, since $S(\cdot)$ is bounded on $\mathcal{X}$, the left-hand side is bounded in absolute value (independently of $T$ ) by some $K_{S}<\infty$. Hence, we have

$$
\int_{0}^{T} \alpha\left(\left\|x^{\star}(t)-\bar{x}^{\star}\right\|\right) d t \leq K_{S}+K_{F} .
$$

Next, using the definition of $\Theta_{\varepsilon, T}$ and the fact that $\alpha$ is of class $\mathcal{K}$, we obtain

$$
\begin{aligned}
\int_{0}^{T} \alpha\left(\left\|x^{\star}(t)-\bar{x}^{\star}\right\|\right) d t \geq \int_{\Theta_{\varepsilon, T}} \alpha\left(\| x^{\star}(t)\right. & \left.-\bar{x}^{\star} \|\right) d t \\
& \geq \alpha(\varepsilon) \mu\left[\Theta_{\varepsilon, T}\right] .
\end{aligned}
$$

Therefore

$$
\mu\left[\Theta_{\varepsilon, T}\right] \leq\left(K_{S}+K_{F}\right) / \alpha(\varepsilon)=: \nu(\varepsilon),
$$

where $\nu(\varepsilon)$ does not depend on $T$.

This result is an extension of a classical result given in [2, Thm. 4.4]. Therein, a turnpike property is proved under the assumption of strict convexity of $F$ and via consideration of a terminal constraint $x(T)=x_{T}$ for the OCP (4). Statements similar to Theorem 1 have been derived for the discrete-time case in $[3,6]$.

Next, we verify that dissipativity implies optimal operation at steady state.

Theorem 2 (Dissipativity $\Rightarrow$ opt. op. at steady-state): If Statement 1 holds at $\bar{x}^{\star}$, then Statement 2 holds, i.e., system (1) is optimally operated at the steady state $\bar{x}^{\star}$.

Proof: For contradiction, assume that there exists an infinite-time admissible pair $\left(x_{\infty}(\cdot), u_{\infty}(\cdot)\right)$ and a sequence 
$\left\{T_{k}\right\}_{k=1}^{\infty}$ with $T_{k+1} \geq T_{k}$ and $T_{k} \rightarrow \infty$ such that

$$
\lim _{k \rightarrow \infty} \frac{1}{T_{k}} J_{T_{k}}\left(x_{0}, u_{\infty}(\cdot)\right) \leq F\left(\bar{x}^{\star}, \bar{u}^{\star}\right)-\sigma
$$

for some $\sigma>0$. Integrating the dissipation inequality (10) and dividing by $T_{k}$ gives

$$
\begin{aligned}
\frac{1}{T_{k}}\left[S\left(x_{\infty}\left(T_{k}\right)\right)\right. & \left.-S\left(x_{\infty}(0)\right)\right] \leq \\
& \leq \frac{1}{T_{k}} J_{T_{k}}\left(x_{0}, u_{\infty}(\cdot)\right)-F\left(\bar{x}^{\star}, \bar{u}^{\star}\right) .
\end{aligned}
$$

Since the storage function $S$ is bounded on $\mathcal{X}$ and $x_{\infty}(t) \in$ $\mathcal{X}$ for all $t \in[0, T]$, the left-hand side of the above inequality converges to zero for $T_{k} \rightarrow \infty$, whereas the right-hand side converges to below $-\sigma<0$, which is a contradiction.

Note that, in the proof of Theorem 2, we never invoked the strict dissipativity term $\alpha\left(\left\|x-\bar{x}^{\star}\right\|\right)$; therefore the following stronger statement holds:

Proposition 1: If system (1) is dissipative (not necessarily strictly) on the set $\mathcal{X} \times \mathcal{U}$ with respect to the steady state $\bar{x}^{\star}$ and the supply rate $w$ from (9), then Statement 2 holds, i.e., the system (1) is optimally operated at the steady state $\bar{x}^{\star}$.

\section{Implications of Turnpike Properties}

Theorem 3 (Turnpike $\Rightarrow$ opt. operated at steady-state): Consider system (1). Suppose that Statement 3 holds at the steady-state $\bar{x}$ and the cost function $F$ does not depend on $u$, i.e., $\frac{\partial F(x, u)}{\partial u}=0$. Then system (1) is optimally operated at the steady state $\bar{x}$.

Proof: Since we assume that $F(x, u)$ is independent of $u$, we simply write $F(x)$ throughout the proof. Fix $x_{0} \in \mathcal{X}_{0}$ and, for contradiction, assume that there exists an infinitetime admissible pair $\left(x_{\infty}(\cdot), u_{\infty}(\cdot)\right)$ and a sequence $\left\{T_{k}\right\}_{k=1}^{\infty}$ with $T_{k+1} \geq T_{k}$ and $T_{k} \rightarrow \infty$ such that

$$
\lim _{k \rightarrow \infty} \frac{1}{T_{k}} J_{T_{k}}\left(x_{0}, u_{\infty}(\cdot)\right) \leq F(\bar{x})-\sigma
$$

for some $\sigma>0$. Next, observe that the turnpike property and Lipschitz continuity of $F(x)$ imply that there exists a function $\gamma(\cdot)$, independent of $T$, such that

$$
\mu\left[\Omega_{\varepsilon, T}\right]<\gamma(\varepsilon)
$$

where

$$
\Omega_{\varepsilon, T}=\left\{t \in[0, T]|| F\left(x_{T}^{\star}(t)\right)-F(\bar{x}) \mid>\varepsilon\right\},
$$

and where $x_{T}^{\star}(\cdot)$ denotes any optimal solution to (4) with horizon $T$. Setting $m:=\min _{x \in \mathcal{X}} F(x)$ and $\Omega_{\varepsilon, T_{k}}^{c}:=$ $\left[0, T_{k}\right] \backslash \Omega_{\varepsilon, T_{k}}$, we have for arbitrary $\varepsilon>0$ :

$$
\begin{array}{rl}
\int_{0}^{T_{k}} & F\left(x_{T_{k}}^{\star}(t)\right) d t=\int_{\Omega_{\varepsilon, T}} F\left(x_{T_{k}}^{\star}(t)\right) d t+\int_{\Omega_{\varepsilon, T}^{c}} F\left(x_{T_{k}}^{\star}(t)\right) d t \\
& \geq m \mu\left[\Omega_{\varepsilon, T_{k}}\right]+\int_{\Omega_{\varepsilon, T}^{c}} F(\bar{x})-\varepsilon d t \\
& =m \mu\left[\Omega_{\varepsilon, T_{k}}\right]+\int_{0}^{T_{k}} F(\bar{x})-\varepsilon d t-\int_{\Omega_{\varepsilon, T_{k}}} F(\bar{x})-\varepsilon d t \\
& =m \mu\left[\Omega_{\varepsilon, T_{k}}\right]+T_{k}(F(\bar{x})-\varepsilon)-\mu\left[\Omega_{\varepsilon, T_{k}}\right](F(\bar{x})-\varepsilon) .
\end{array}
$$

Since $\mu\left[\Omega_{\varepsilon, T_{k}}\right]<\gamma(\varepsilon)$ independently of $T_{k}$, dividing by $T_{k}$ and letting $k \rightarrow \infty$ gives:

$$
\lim _{k \rightarrow \infty} \frac{1}{T_{k}} \int_{0}^{T_{k}} F\left(x_{T_{k}}^{\star}(t)\right) d t \geq F(\bar{x})-\varepsilon .
$$

Selecting $\varepsilon<\sigma$, we arrive at a contradiction to (14) since the pair $\left(x_{\infty}(\cdot), u_{\infty}(\cdot)\right)$ truncated to $\left[0, T_{k}\right]$ is admissible for the OCP (4) for any $T_{k} \geq 0$.

The following corollary says that a turnpike can occur only at an optimal steady state:

Corollary 1 (Turnpikes only at optimal steady state):

Let the assumptions of Theorem 3 hold. Then there exists a $\bar{u} \in \mathcal{U}$ such that $(\bar{x}, \bar{u})=\left(\bar{x}^{\star}, \bar{u}^{\star}\right)$, i.e. $(\bar{x}, \bar{u})$ is optimal in (5).

Proof: Follows from Theorem 3 and Lemma 1.

Remark 3: The preceding corollary is a consequence of the turnpike definition used in this paper, which implies asymptotic reachability of $\bar{x}$ from all $x_{0} \in \mathcal{X}_{0}$ (see Remark 1). If instead a local definition of turnpike is used, analogous local results can be established.

One might wonder whether or not the consideration of purely state-dependent cost functions $F$ is restricting. There are two answers to this question: (a) one augments the dynamics (1) by considering the inputs $u$ as state variables and using $\dot{u}$ as new input variables; (b) one may change the definition of the turnpike property. If one changes (8) such that instead of $\left\|x^{\star}\left(t, x, u^{\star}(\cdot)\right)-\bar{x}\right\|$ the distance $\left\|\left(x^{\star}\left(t, x, u^{\star}(\cdot)\right), u^{\star}(t)\right)-(\bar{x}, \bar{u})\right\|$ is considered, then Theorem 3 can be extended to general Lipschitz continuous cost functions $F: \mathcal{X} \times \mathcal{U} \rightarrow \mathbb{R}$.

The next result shows that augmenting the system by considering $\dot{u}(t)$ as new inputs preserves dissipativity.

Lemma 3: Suppose, that the system (1) is dissipative on $\mathcal{D} \subseteq \mathcal{X} \times \mathcal{U}$ with respect to a steady state $\bar{x} \in \overline{\mathcal{X}}, f(\bar{x}, \bar{u})=$ 0 . Then, the augmented system

$$
\dot{z}=g(z, v)=\left(\begin{array}{c}
f(x, u) \\
v
\end{array}\right), \quad z=(x, u)
$$

is dissipative with respect to the steady state $\bar{z}=(\bar{x}, \bar{u})$.

Proof: Consider the candidate storage function $\Sigma$ : $\mathbb{R}^{n_{x}+n_{u}} \rightarrow \mathbb{R}, \Sigma(z)=\Sigma(x, u)$. Evaluation of $\Sigma$ along the trajectories of the augmented system (15) yields

$$
\frac{\partial \Sigma}{\partial z} g(z, v)=\left(\frac{\partial \Sigma}{\partial x}, \frac{\partial \Sigma}{\partial u}\right)\left(\begin{array}{c}
f(x, u) \\
v
\end{array}\right)=\frac{\partial \Sigma}{\partial x} f(x, u)+\frac{\partial \Sigma}{\partial u} v .
$$

Dissipativity of (1) with respect to a steady state $\bar{x}$ : $f(\bar{x}, \bar{u})=0$ implies $\frac{\partial S}{\partial x} f(x, u) \leq w(x, u)$. Thus, we obtain that the supply rate $w(z)=w(x, u)$ and the storage function $\Sigma(z)=S(x)$ satisfy the dissipation inequality (10).

Thus we conclude that the assumption of purely statedependent cost functions is not overly restrictive.

\section{ExAmple: Chemical ReActor}

To illustrate the results from the last section we consider a Van de Vusse reactor as an example, [15]. In a continuously stirred tank reactor, three endothermal chemical reactions $A \stackrel{k_{1}}{\rightarrow} B \stackrel{k_{2}}{\rightarrow} C$ and $2 A \stackrel{k_{3}}{\rightarrow} D$ take place. A partial model 
of the reactor, including the concentration of species $A$ and $B, c_{A}, c_{B}$ in $\mathrm{mol} / \mathrm{l}$ and the reactor temperature $\vartheta$ in ${ }^{\circ} \mathrm{C}$ as state variables, reads

$$
\begin{aligned}
\dot{c}_{A} & =r_{A}\left(c_{A}, \vartheta\right)+\left(c_{i n}-c_{A}\right) u_{1} \\
\dot{c}_{B} & =r_{B}\left(c_{A}, c_{B}, \vartheta\right)-c_{B} u_{1} \\
\dot{\vartheta} & =h\left(c_{A}, c_{B}, \vartheta\right)+\alpha\left(u_{2}-\vartheta\right)+\left(\vartheta_{i n}-\vartheta\right) u_{1},
\end{aligned}
$$

where

$$
\begin{aligned}
r_{A}\left(c_{A}, \vartheta\right) & =-k_{1}(\vartheta) c_{A}-2 k_{3}(\vartheta) c_{A}^{2} \\
r_{B}\left(c_{A}, c_{B}, \vartheta\right) & =k_{1}(\vartheta) c_{A}-k_{2}(\vartheta) c_{B} \\
h\left(c_{A}, c_{B}, \vartheta\right)= & -\delta\left(k_{1}(\vartheta) c_{A} \Delta H_{A B}+k_{2}(\vartheta) c_{B} \Delta H_{B C}\right. \\
& \left.\quad+2 k_{3}(\vartheta) c_{A}^{2} \Delta H_{A D}\right) \\
k_{i}(\vartheta)= & k_{i 0} \exp \frac{-E_{i}}{\vartheta+\vartheta_{0}}, \quad i=1,2,3
\end{aligned}
$$

The system parameters can be found in [15]. The inputs $u_{1}, u_{2}$ are the normalized flow rate of $A$ through the reactor in $1 / \mathrm{h}$ and the temperature in the cooling jacket in ${ }^{\circ} \mathrm{C}$. The states and inputs are subject to the constraints

$$
\begin{array}{lll}
c_{A} \in[0,6] \frac{\mathrm{mol}}{l} & c_{B} \in[0,4] \frac{\mathrm{mol}}{l} & \vartheta \in[70,200]^{\circ} \mathrm{C} \\
u_{1} \in[3,35] \frac{1}{h} & u_{2} \in[0,150]^{\circ} \mathrm{C} . &
\end{array}
$$

We consider the problem of maximizing the production rate of $c_{B}$; thus we specify the cost function $F$ in (3) and (5) as

$$
F\left(c_{B}, u_{1}\right)=-\beta c_{B} u_{1}, \quad \beta>0 .
$$

\section{A. Numerical Verification of Dissipativity}

To numerically verify dissipativity, we approximate the exponential term $k_{i}(\theta)$ by its fourth-order Taylor expansion at $\vartheta=110^{\circ} \mathrm{C}$. This yields a reasonable approximation of $k_{i}(\theta)$ over the box $[70,200]$. This way the system dynamics become polynomial and a polynomial storage function can be sought using sum-of-squares (SOS) programming. Indeed, by choosing a quadratic class $\mathcal{K}$ function $\alpha:=\bar{\alpha}\left\|x-\bar{x}^{\star}\right\|^{2}$, where $\bar{\alpha}>0$ is an optimization variable, all data in the dissipation inequality (11) become polynomial. We solve the following optimization problem:

$$
\underset{\bar{\alpha} \in[0,1], S(\cdot) \in \mathbb{R}[x]}{\operatorname{maximize}} \bar{\alpha}
$$

subject to $\forall(x, u) \in \mathcal{X} \times \mathcal{U}$

$$
w(x, u)-\bar{\alpha}\left\|x-\bar{x}^{\star}\right\|^{2}-\frac{\partial S}{\partial x} f(x, u) \geq 0,
$$

where $\mathbb{R}[x]$ denotes the ring of polynomials. The nonnegativity constraint (19b) is replaced by a sufficient SOS constraint; here we used the standard Putinar condition [13] imposed for each of the four vertices of $\mathcal{U}$ separately (this is possible since $u$ enters the dynamics affinely and $\mathcal{U}$ is convex). This leads to a semidefinite programming problem (SDP), which is solved using SeDuMi [12]. Details of sum-of-squares programming are omitted for brevity; see, e.g., [9] for a general treatment or [5] for an application of SOS programming to dissipativity problems. The strict dissipativity constant $\bar{\alpha}$ is constrained to $[0,1]$ for numerical reasons.
The optimal steady state $\bar{x}^{\star}=[2.1756,1.1049,128.53]^{T}$, $\bar{u}^{\star}=[35,142.76]^{T}$ is computed using an SQP-method from Matlab's function fmincon, and its global optimality is verified using Gloptipoly [7].

Seeking a polynomial storage function of degree five using sum-of-squares programming results in a feasible problem with $\bar{\alpha}=1$, thus verifying strict dissipativity with respect to $\bar{x}^{\star}$ on $\mathcal{X} \times \mathcal{U}$.

\section{B. Numerical Verification of Turnpike Properties}

In order to numerically show turnpike properties, we solve the following OCP

$$
\begin{gathered}
\underset{u(\cdot)}{\operatorname{minimize}} \int_{0}^{T}-\beta c_{B}(t) u_{1}(t) d t \\
\text { subject to (16) and (17). }
\end{gathered}
$$

To foster comparability with the numerically obtained storage function, the Arrhenius terms (16g) are again approximated by the fourth-order Taylor series developed at $\vartheta=$ $110^{\circ} \mathrm{C}$. OCP (20) is solved for two initial conditions with a piecewise-constant input parametrization via a direct multiple shooting implementation [8]. For the initial condition $\left.c_{A}(0), c_{B}(0), \vartheta(0)\right)=(1.5,1.2,140)$ we consider an optimization horizon of $T=0.5$; for $\left.c_{A}(0), c_{B}(0), \vartheta(0)\right)=$ $(5.1,0,100)$ we use $T=0.25$. The results are depicted in Fig. 2.

The plots in the upper part of Fig. 2 show the trajectories of states $c_{A}, c_{B}$ and $\vartheta$ and their optimal steady-state values. The dynamic trajectories obtained via OCP (20) are plotted in blue or green depending on the considered horizon length. The optimal steady-state values are plotted in red. Clearly, the optimal solutions converge rapidly to the optimal steady state and diverge close to the end of the horizon. In other words, the optimal solutions to (20) exhibit the turnpike property.

The lower part of Fig. 2 depicts the inputs $u_{1}, u_{2}$ (middle and right plot). Note that the input $u_{1}$ is always at its upper limit. The left plot illustrates the strict dissipation inequality (11). To this end we define

$$
\delta(t)=\left.\left(\frac{\partial S}{\partial x} f(x, u)+\alpha\left(\left\|x-\bar{x}^{\star}\right\|\right)-w(x, u)\right)\right|_{\substack{x=x(t) \\ u=u(t)}},
$$

where $\alpha\left(\left\|x-\bar{x}^{\star}\right\|\right), w(x, u)$ and the storage $S(x)$ are the ones computed in Section IV-A. Note that $\delta(t) \leq 0$ implies that the strict dissipation inequality (11) is satisfied.

In Fig. 2 (lower part, left side) $\delta(t)$ is plotted along the optimal solutions to (20). We see that the dissipation inequality (11) is satisfied for both cases.

\section{CONClusions}

This paper has investigated the relation between dissipativity properties of optimal control problems, turnpike properties of the optimal solutions, and optimal operation at steady state over an infinite horizon. We extended discretetime results to show that dissipativity implies (a) optimal operation at steady state and (b) a turnpike property of optimal solutions. Furthermore, we showed that turnpike properties 

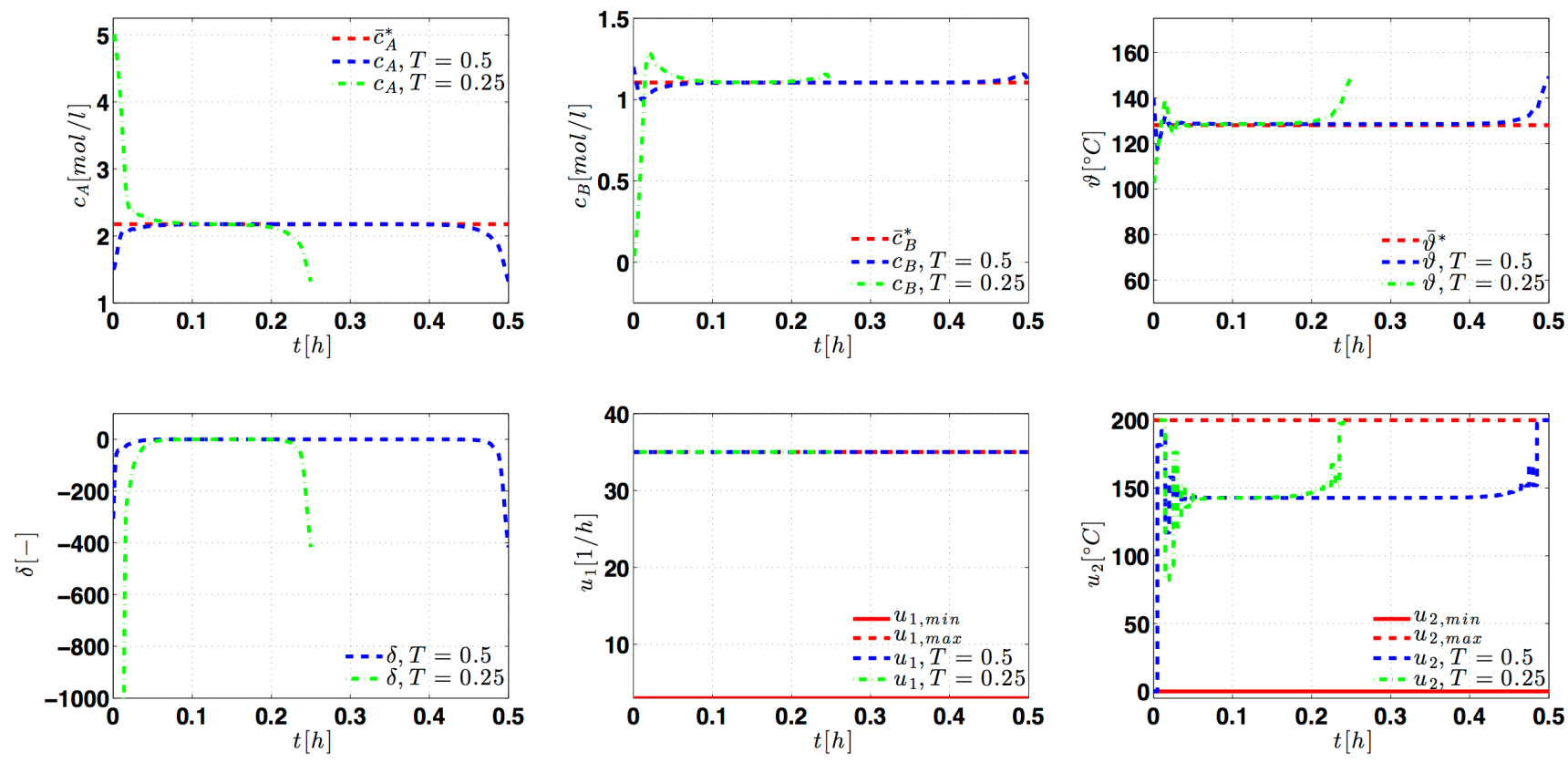

Fig. 2. Simulation results for the Van de Vusse reactor (16).

of optimal solutions imply optimal operation at steady state. Finally, we demonstrated by means of a numerical example that, for polynomial systems, dissipativity of optimal control problems can be verified via sum-of-squares programming.

Future work will consider the establishment of additional relations between dissipativity, turnpikes and optimal operation at steady state.

\section{BIBLIOGRAPHY}

[1] D. Angeli, R. Amrit, and J. Rawlings. "On average performance and stability of economic model predictive control". In: IEEE Trans. Automat. Contr. 57:7 (2012), pp. 1615-1626.

[2] D.A. Carlson, A. Haurie, and A. Leizarowitz. Infinite Horizon Optimal Control: Deterministic and Stochastic Systems. 2nd. Springer-verlag, 1991.

[3] T. Damm, L. Grüne, M. Stieler, and K. Worthmann. "An exponential turnpike theorem for dissipative optimal control problems". In: SIAM Journal on Control and Optimization 52:3 (2014), pp. 1935-1957.

[4] M. Diehl, R. Amrit, and J.B. Rawlings. "A Lyapunov function for economic optimizing model predictive control". In: IEEE Trans. Automat. Contr. 56:3 (2011), pp. 703-707.

[5] C. Ebenbauer and F. Allgöwer. "Analysis and design of polynomial control systems using dissipation inequalities and sum of squares". In: Computers and Chemical Engineering 30:3 (2006), pp. 1590-1602.

[6] L. Grüne. "Economic receding horizon control without terminal constraints". In: Automatica 49:3 (2013), pp. 725 -734. ISSN: 0005-1098.

[7] D. Henrion, J.B. Lasserre, and J. Löfberg. "Gloptipoly 3: moments, optimization and semidefinite programming". In: Optimization Methods and Software 24 (2009), pp. 761-779.

[8] B. Houska, H.J. Ferreau, and M. Diehl. "ACADO toolkit - an open-source framework for automatic control and dynamic optimization". In: Optimal Control Applications and Methods 32:3 (2011), pp. 298-312.
[9] J.B. Lasserre. Moments, Positive Polynomials and Their Applications, Imperial College Press, 2009. ISBN: 978-1-84816-445-1.

[10] E.B. Lee and L. Markus. Foundations of Optimal Control Theory. The SIAM series in applied mathematics. John Wiley \& Sons New York, London, Sydney, 1967.

[11] M.A. Müller, D. Angeli, and F. Allgöwer. "On convergence of averagely constrained economic mpc and necessity of dissipativity for optimal steady-state operation". In: Proceedings of the American Control Conference. 2013.

[12] I. Pólik, T. Terlaky, and Y. Zinchenko. "Sedumi: a package for conic optimization". In: IMA Workshop on Optimization and Control. 2007.

[13] M. Putinar. "Positive polynomials on compact semi-algebraic sets". In: Indiana University Mathematics Journal, 42 (1993), pp. 969-984.

[14] J. Rawlings and R. Amrit. "Optimizing process economic performance using model predictive control". In: Nonlinear Model Predictive Control - Towards New Challenging Applications. Ed. by L. Magni, D. Raimondo, and F. Allgöwer. Vol. 384. Lecture Notes in Control and Information Sciences. Springer Berlin, 2009, pp. 119-138.

[15] R. Rothfuß, J. Rudolph, and M. Zeitz. "Flatness based control of a nonlinear chemical reactor model". In: Automatica 32 (1996), pp. 1433-1439.

[16] R. Vinter. Optimal Control. Springer, 2010.

[17] L. Würth, J. Rawlings, and W. Marquardt. "Economic dynamic real-time optimization and nonlinear model-predictive control on infinite horizons". In: ADCHEM 2009 (International Symposium on Advanced Control of Chemical Processes), Istanbul, Turkey, 12-15.07.2009. 2009.

[18] A.J. Zaslavski. Turnpike Properties in the Calculus of Variations and Optimal Control. Vol. 80. Springer, 2006. 